\title{
Dual jet bundles, Hamiltonian systems and connections
}

\author{
Olga Rossi and David Saunders
}

December 3, 2013

\begin{abstract}
We define Hamilton-De Donder systems on a dual jet bundle, and show that they are variational in a general sense. We explore the relationship between these systems and Ehresmann connections. We also consider regularity conditions for such systems and show that, when regular, they arise from Lagrangian systems on the original jet bundle.

MSC: 58E30, 58A20, 58J72

Keywords: affine, Lagrangian, Hamiltonian, Hamilton-De Donder, Ehresmann connection
\end{abstract}

\section{Introduction}

In this paper we consider those second-order partial differential equations arising as the variational equations of a regular Lagrangian. We explain the Hamiltonian formalism for these equations: more precisely, we examine the geometry of the Hamilton-De Donder equations for a regular Lagrangian, both on a jet bundle and also on the corresponding dual jet bundle, and we compare the two approaches. To each regular Lagrangian system we associate a Hamiltonian system, and we establish conditions under which the reverse correspondence holds.

The main approach of the paper involves the use of connections related to the Hamiltonian systems and the corresponding second-order partial differential equations. Our main results concern the construction of connections satisfying the condition that every integral section is an extremal of the variational problem, and conversely we show that every extremal can be embedded in a connection of this kind. We give an explicit description of all such connections, and in this way we can describe all the local solutions of the Euler-Lagrange equations in terms of the related connections.

The point of view we have adopted is a generalization of the Hamiltonian formalism of symplectic geometry: in that case, with a single independent variable, the extremals of a regular variational problem can be expressed as the integral curves of a vector field [13]. It is well known that there are several ways of formulating a generalization to the case of several independent variables, as needed for the study of PDEs and field theory, and our approach uses a multisymplectic formulation on dual jet bundles [9]. We concentrate on the most familiar case of the Hamilton-De Donder equations of a regular Lagrangian, and we also investigate the same equations using the geometric framework originated by 
Goldschmidt and Sternberg [8] (following De Donder [4]). We see that there is a strong parallel between these two approaches and the corresponding geometric structures. Some selected references are $[1,2,3,5,6,7,10,11,14]$.

The structure of this paper is as follows. In Section 2 we review the affine duality structure of jet bundles in $n$ independent variables and give an invariant definition of those systems of first-order partial differential equations that may be described as being of Hamilton-De Donder type and are therefore generated by a Hamiltonian. We show that such equations are variational, and give conditions for a general system of first-order PDEs in the correct format to be Hamilton-De Donder equations. This approach, which we subsequently refine in Section 5, also gives us the solution of the inverse variational problem in a Hamiltonian formulation, so that we can see when a given system of first order PDEs is a Hamilton-De Donder system related to a regular Lagrangian, and it also allows us to reconstruct the corresponding Lagrangian system of second-order variational PDEs.

In Section 3 we move on to consider the compatibility relationship between HamiltonDe Donder equations and the equations arising from an Ehresmann connection, showing that a global compatible connection always exists, and that every solution of the Hamilton-De Donder equations is also, locally, an integral section of some compatible connection; this allows us to give a definition of a completely integrable Hamilton-De Donder system. In Section 4 we compare this to the theory of Goldschmidt and Sternberg, where Hamilton-De Donder equations are defined on a jet bundle rather than its dual, and the corresponding connections are semispray connections. We are able to give an explicit formula for the family of connections satisfying these conditions. Finally in Section 5 we consider both Lagrangian and Hamiltonian structures together, showing that if a Lagrangian is regular in the usual sense then there is a corresponding Hamiltonian structure, and that conversely if the Hamiltonian is 'regular' then it arises from a Lagrangian; we also revisit the inverse problem in this context.

\section{Hamiltonian systems on jet bundles}

\subsection{Dual jet bundles}

Let $\pi: Y \rightarrow X$ be a fibred manifold with $\operatorname{dim} X=n, \operatorname{dim} Y=n+m$ and let $\pi_{1}$ : $J^{1} Y \rightarrow X$ be its first jet bundle over $X$. The manifold $J^{1} Y$ is also the total space of an affine bundle $\pi_{1,0}: J^{1} Y \rightarrow Y$ modelled on the vector bundle $V_{X} Y \otimes \pi^{*} T^{*} X \rightarrow Y$, where $V_{X} Y \rightarrow Y$ is the bundle of vectors tangent to $Y$ and vertical over $X$. We shall use the notation $J_{y}^{1} Y$ for a fibre $\pi_{1,0}^{-1}(y) \subset J^{1} Y$ where $y \in Y$, and put $x=\pi(y) \in X$.

To see how this affine structure arises, let $\gamma$ be a local section of $\pi$ satisfying $\gamma(x)=y$.

The equivalence class of local sections $\gamma$ defining the jet $j_{x}^{1} \gamma$ is the same as the equivalence class defining the tangent map $(T \gamma)_{x}: T_{x} X \rightarrow T_{y} Y$, so that we may identify $j_{x}^{1} \gamma \in J_{y}^{1} Y$ with

$$
(T \gamma)_{x} \in \operatorname{Hom}\left(T_{x} X, T_{y} Y\right) \cong T_{y} Y \otimes T_{x}^{*} X
$$

where we consider the latter space as a subspace of the tensor space $T_{y} Y \otimes T_{y}^{*} Y$. Taking 
$\left(x^{i}, y^{\sigma}\right), 1 \leq i \leq n, 1 \leq \sigma \leq m$ as local fibred coordinates on $Y$, with $\left(x^{i}, y^{\sigma}, y_{j}^{\sigma}\right)$ as the associated coordinates on $J^{1} Y$, the jet $j_{x}^{1} \gamma$ corresponds to the tensor

$$
\left(\frac{\partial}{\partial x^{i}}+y_{i}^{\sigma}\left(j_{x}^{1} \gamma\right) \frac{\partial}{\partial y^{\sigma}}\right) \otimes d x^{i}
$$

at $y \in Y$, where the coefficient of $\partial / \partial x^{j}$ is $\delta_{i}^{j}$ because $\gamma$ is a local section.

Every affine space of dimension $m$ is associated with a vector space of dimension $m+1$, its extended dual, whose elements are the real-valued affine maps on the space. Applying this construction to the fibres $J_{y}^{1} Y$ of the affine bundle $\pi_{1,0}$ gives the manifold $J^{\dagger} Y$, the extended dual of $J^{1} Y$. If $\phi \in J^{\dagger} Y$ and $\phi: J_{y}^{1} Y \rightarrow \mathbb{R}$ we put $\pi_{1,0}^{\dagger}(\phi)=y$, and then $\pi_{1,0}^{\dagger}: J^{\dagger} Y \rightarrow Y$ becomes a vector bundle.

Each fibre $J_{y}^{\dagger} Y$ of the extended dual is a vector space containing a distinguished subspace of constant maps. The quotient space will be denoted $J_{y}^{*} Y$, and the union of all the quotient spaces forms another vector bundle, the reduced dual $J^{*} Y$. We shall let $\rho: J^{\dagger} Y \rightarrow J^{*} Y$ denote the quotient map, and $\pi_{1,0}^{*}: J^{*} Y \rightarrow Y$ denote the induced map. The composition $\pi \circ \pi_{1,0}^{*}: J^{*} Y \rightarrow X$ will be denoted by $\tau$. We can see how all these maps fit together in the following diagram.

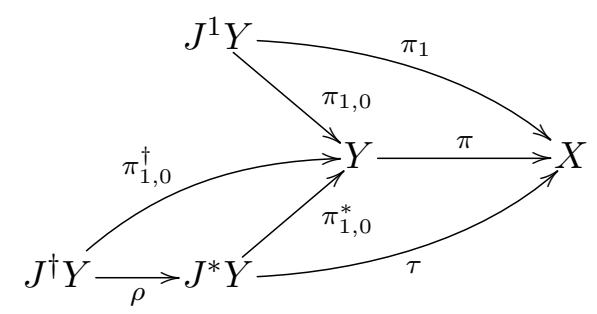

If $y$ is in the domain of the chart $\left(x^{i}, y^{\sigma}\right)$, let $y_{i(y)}^{\sigma}$ be the restrictions of the induced coordinate functions $y_{i}^{\sigma}$ to the fibre $J_{y}^{1} Y$, so that $y_{i(y)}^{\sigma}$ are affine functions and are elements of the fibre $J_{y}^{\dagger} Y$. The constant function $1_{(y)}: J_{y}^{1} Y \rightarrow \mathbb{R}$ is also an affine function, and together the functions $\left(1_{(y)}, y_{i(y)}^{\sigma}\right)$ form a basis for the vector space $J_{y}^{\dagger} Y$. Denote the dual basis of $\left(J_{y}^{\dagger} Y\right)^{*}$ by $\left(P_{(y)}, P_{\sigma(y)}^{i}\right)$; then the functions $P, P_{\sigma}^{i}$ defined locally on $J^{\dagger} Y$ by these fibre functions are smooth, and the functions $\left(x^{i}, y^{\sigma}, P, P_{\sigma}^{i}\right)$ form an adapted coordinate system. Similarly the functions $\left(x^{i}, y^{\sigma}, P_{\sigma}^{i}\right)$ are coordinates on the reduced dual $J^{*} Y$.

Now suppose that there is a given volume $n$-form $\omega_{0}$ on $X$ such that the coordinates $x^{i}$ satisfy $\omega_{0}=d x^{1} \wedge d x^{2} \wedge \cdots \wedge d x^{n}$, and consider the bundle of cotangent $n$-vectors

$$
\bigwedge_{1}^{n} T^{*} Y=\left\{\theta \in \bigwedge^{n} T^{*} Y: \xi, \eta \in V_{X} Y \Rightarrow i_{\xi} i_{\eta} \theta=0\right\},
$$

so that an element $\theta$ of this bundle may be written in the coordinates $\left(x^{i}, y^{\sigma}\right)$ as

$$
\theta=\theta_{0} \omega_{0}+\theta_{\sigma}^{i} d y^{\sigma} \wedge \omega_{i}
$$

where $\omega_{i}=i_{\partial / \partial x^{i}} \omega_{0}$. We may identify this bundle with the extended dual in the following way. Take $\theta \in \bigwedge_{1}^{n} T_{y}^{*} Y$, and let $\gamma$ be any local section satisfying $\gamma(x)=y$. The pull-back 
cotangent $n$-vector $\gamma^{*}(\theta) \in \bigwedge^{n} T_{x}^{*} X$ depends only on the value and first derivatives of $\gamma$ at $x$, and so the function

$$
\widetilde{\theta}: J_{y}^{1} Y \rightarrow \mathbb{R}, \quad \widetilde{\theta}\left(j_{x}^{1} \gamma\right) \omega_{0}=\gamma^{*}(\theta)
$$

is well-defined. In coordinates

$$
\widetilde{\theta}\left(j_{x}^{1} \gamma\right)=\theta_{0}+\left.\theta_{\sigma}^{i} \frac{d \gamma^{\sigma}}{d x^{i}}\right|_{x}=\theta_{0}+\theta_{\sigma}^{i} y_{i}^{\sigma}\left(j_{x}^{1} \gamma\right)
$$

showing that $\widetilde{\theta}$ is an affine function. This correspondence is a linear isomorphism of the fibres, under which the basis $\left(1_{(y)}, y_{i(y)}^{\sigma}\right)$ of $J_{y}^{\dagger} Y$ corresponds to the basis $\left(\left.\omega_{0}\right|_{y},\left.d y^{\sigma} \wedge \omega_{i}\right|_{y}\right)$ of $\bigwedge_{1}^{n} T_{y}^{*} Y$, and gives rise to a diffeomorphism $J^{\dagger} Y \cong \bigwedge_{1}^{n} T^{*} Y$. The canonical $n$-form

$$
\Theta=P \omega_{0}+P_{\sigma}^{i} d y^{\sigma} \wedge \omega_{i}
$$

on $\bigwedge_{1}^{n} T^{*} Y$ may therefore be regarded as an $n$-form on the extended dual $J^{\dagger} Y$, and similarly the $(n+1)$-form $\Omega=d \Theta$ may be regarded as an $(n+1)$-form on $J^{\dagger} Y$. As $\Omega$ is a multisymplectic form, $\left(J^{\dagger} Y, \Omega\right)$ becomes a multisymplectic manifold. Note that, owing to the affine structure, the multisymplectic form $\Omega$ is canonical for the manifold $J^{\dagger} Y$, although it is not the only multisymplectic form on $J^{\dagger} Y$. For reasons which will become clear later on, we shall also call $\Omega$ the Hamilton-De Donder multisymplectic form.

\subsection{Hamilton-De Donder systems}

Any local section $\mathrm{h}$ of the quotient projection $\rho: J^{\dagger} Y \rightarrow J^{*} Y$, with domain $U \subset J^{*} Y$, gives rise to a local 'Hamilton-De Donder system' in the following way. Consider the pullbacks $\Theta_{\mathrm{h}}=\mathrm{h}^{*} \Theta, \Omega_{\mathrm{h}}=\mathrm{h}^{*} \Omega$ of the canonical forms to $U$, and let $\mathcal{H}=-(P \circ \mathrm{h})$ be (minus) the nontrivial component of the section $\mathrm{h}$ in the given coordinates, so that $\mathcal{H}$ is a function defined on $U$; then

$$
\Theta_{\mathrm{h}}=-\mathcal{H} \omega_{0}+P_{\sigma}^{i} d y^{\sigma} \wedge \omega_{i}, \quad \Omega_{\mathrm{h}}=-d \mathcal{H} \wedge \omega_{0}+d P_{\sigma}^{i} \wedge d y^{\sigma} \wedge \omega_{i} .
$$

We now define the Hamilton-De Donder system $\mathcal{D}_{\mathrm{h}}$ to be the exterior differential system on $U$ generated by the family of $n$-forms $i_{\xi} \Omega_{\mathrm{h}}$, where $\xi$ runs over all vector fields on $U$ that are vertical over $X$. Taking for $\xi$ each of the basis vector fields $\left(\partial / \partial y^{\sigma}, \partial / \partial P_{\sigma}^{i}\right)$, we see that locally

$$
\mathcal{D}_{\mathrm{h}}=\operatorname{span}\left\{\eta_{\sigma}, \eta_{i}^{\sigma}\right\}, \quad \eta_{\sigma}=-\frac{\partial \mathcal{H}}{\partial y^{\sigma}} \omega_{0}-d P_{\sigma}^{i} \wedge \omega_{i}, \quad \eta_{i}^{\sigma}=-\frac{\partial \mathcal{H}}{\partial P_{\sigma}^{i}} \omega_{0}+d y^{\sigma} \wedge \omega_{i}
$$

If $\psi$ is a local section of $\tau: J^{*} Y \rightarrow X$ then $\psi$ annihilates $\mathcal{D}_{\mathrm{h}}$ (that is, $\psi$ is a solution of the Hamilton-De Donder exterior differential system) if and only if $\psi$ satisfies the equation (2.2) $\psi^{*}\left(i_{\xi} \Omega_{\mathrm{h}}\right)=0 \quad$ for every vector field $\xi$ on $J^{*} Y$, vertical over $X$.

In coordinates,

$$
-\frac{\partial \mathcal{H}}{\partial y^{\sigma}}-\frac{\partial \psi_{\sigma}^{i}}{\partial x^{i}}=0, \quad-\frac{\partial \mathcal{H}}{\partial P_{\sigma}^{i}}+\frac{\partial \psi^{\sigma}}{\partial x^{i}}=0
$$


for the components $\psi^{\sigma}=y^{\sigma} \circ \psi$ and $\psi_{\sigma}^{i}=P_{\sigma}^{i} \circ \psi$ of $\psi$. (The apparently perverse choice of sign for these equations is needed to ensure that, as we shall see in the next section, the equations are strictly variational without needing a multiplier.) We shall refer to these equations as Hamilton equations of De Donder type, or as Hamilton-De Donder equations. The images of solutions $\psi$ are immersed $n$-dimensional submanifolds of the $(n+m+n m)$-dimensional manifold $J^{*} Y$.

Remark 2.1. It is easy to check that condition (2.2) is equivalent to

(2.4) $\psi^{*}\left(i_{\xi} \Omega_{\mathrm{h}}\right)=0 \quad$ for every vector field $\xi$ on $J^{*} Y$.

So considering the exterior differential system $\tilde{\mathcal{D}}_{\mathrm{h}}$ generated by $n$-forms $i_{\xi} \Omega_{\mathrm{h}}$ where $\xi$ runs over all vector fields on $U$, we can see that $\mathcal{D}_{\mathrm{h}}$ and $\tilde{\mathcal{D}}_{\mathrm{h}}$ have the same set of solutions, even though $\mathcal{D}_{\mathrm{h}} \varsubsetneqq \tilde{\mathcal{D}}_{\mathrm{h}}$.

Theorem 2.2. The ideal generated by $\mathcal{D}_{\mathrm{h}}$ is a differential ideal, and its Cauchy distribution is trivial.

Proof. As

$$
d x^{j} \wedge \eta_{\sigma}=d P_{\sigma}^{j} \wedge \omega_{0}, \quad d x^{i} \wedge \eta_{i}^{\sigma}=-n d y^{\sigma} \wedge \omega_{0},
$$

we obtain

$$
\begin{aligned}
d \eta_{\sigma} & =-\frac{\partial^{2} \mathcal{H}}{\partial y^{\sigma} \partial y^{\nu}} d y^{\nu} \wedge \omega_{0}-\frac{\partial^{2} \mathcal{H}}{\partial y^{\sigma} \partial P_{\nu}^{j}} d P_{\nu}^{j} \wedge \omega_{0} \\
& =\frac{1}{n} \frac{\partial^{2} \mathcal{H}}{\partial y^{\sigma} \partial y^{\nu}} d x^{i} \wedge \eta_{i}^{\nu}-\frac{\partial^{2} \mathcal{H}}{\partial y^{\sigma} \partial P_{\nu}^{j}} d x^{j} \wedge \eta_{\nu} \\
d \eta_{i}^{\sigma} & =-\frac{\partial^{2} \mathcal{H}}{\partial P_{\sigma}^{i} \partial y^{\nu}} d y^{\nu} \wedge \omega_{0}-\frac{\partial^{2} \mathcal{H}}{\partial P_{\sigma}^{i} \partial P_{\nu}^{j}} d P_{\nu}^{j} \wedge \omega_{0} \\
& =\frac{1}{n} \frac{\partial^{2} \mathcal{H}}{\partial P_{\sigma}^{i} \partial y^{\nu}} d x^{i} \wedge \eta_{i}^{\nu}-\frac{\partial^{2} \mathcal{H}}{\partial P_{\sigma}^{i} \partial P_{\nu}^{j}} d x^{j} \wedge \eta_{\nu}
\end{aligned}
$$

proving the first of the assertions. We also observe that $\zeta$ belongs to the Cauchy distribution only if $i_{\zeta} \eta_{\sigma}=0$ and $i_{\zeta} \eta_{i}^{\sigma}=0$, showing that $\zeta=0$.

\subsection{Variational principles for Hamiltonian systems}

Our aim now is to look for a variational principle whose extremals are local sections $\psi$ of the fibred manifold $\tau: J^{*} Y \rightarrow X$ that are precisely the solutions of the Hamilton-De Donder equations (2.3). These equations are system of first order PDEs over the fibred manifold $\tau: J^{*} Y \rightarrow X$ and define a submanifold of $J^{1}\left(J^{*} Y\right)$ projecting to $U \subset J^{*} Y$, so that we can tackle the problem directly by applying the Helmholtz conditions to the equations. 
Given fibred coordinates $\left(x^{i}, z^{I}\right)=\left(x^{i}, y^{\sigma}, P_{\sigma}^{i}\right)$ on $J^{*} Y$, denote the associated coordinates on $J^{1}\left(J^{*} Y\right)$ by $\left(x^{i}, z^{I}, z_{\mid j}^{I}\right)=\left(x^{i}, y^{\sigma}, P_{\sigma}^{i}, y_{\mid j}^{\sigma}, P_{\sigma \mid j}^{i}\right)$ so that equations (2.3) take the form

$$
\left(-\frac{\partial \mathcal{H}}{\partial y^{\sigma}}-P_{\sigma \mid i}^{i}\right) \circ j^{1} \psi=0, \quad\left(-\frac{\partial \mathcal{H}}{\partial P_{\sigma}^{i}}+y_{\mid i}^{\sigma}\right) \circ j^{1} \psi=0 .
$$

Writing $E$ for the $(n+1)$-form

$$
E=E_{J} d z^{J} \wedge \omega_{0}=\left(-\frac{\partial \mathcal{H}}{\partial y^{\sigma}}-P_{\sigma \mid i}^{i}\right) d y^{\sigma} \wedge \omega_{0}+\left(-\frac{\partial \mathcal{H}}{\partial P_{\sigma}^{i}}+y_{\mid i}^{\sigma}\right) d P_{\sigma}^{i} \wedge \omega_{0}
$$

and substituting into the Helmholtz conditions

$$
\frac{\partial E_{I}}{\partial z_{\mid i}^{J}}+\frac{\partial E_{J}}{\partial z_{\mid i}^{I}}=0, \quad \frac{\partial E_{I}}{\partial z^{J}}-\frac{\partial E_{J}}{\partial z^{I}}+d_{i} \frac{\partial E_{J}}{\partial z_{\mid i}^{I}}=0
$$

we see that the conditions are satisfied, showing that the Hamilton-De Donder equations are variational. A corresponding local Lagrangian can then be computed using the Tonti formula

$$
\mathcal{L}=z^{I} \int_{0}^{1}\left(E_{I} \circ \chi\right) d u \quad \text { where } \chi\left(u,\left(x^{i}, z^{J}, z_{\mid i}^{J}\right)\right)=\left(x^{i}, u z^{J}, u z_{\mid i}^{J}\right)
$$

defined on a proper star-shaped neighborhood of each point in $\tau_{1,0}^{-1}(U)$. Using the explicit formula for the functions $E_{I}$ then gives

$$
\begin{aligned}
\mathcal{L}_{\mathrm{h}} & =y^{\sigma} \int_{0}^{1}\left(-\frac{\partial \mathcal{H}}{\partial y^{\sigma}}-P_{\sigma \mid i}^{i}\right) \circ \chi d u+P_{\sigma}^{i} \int_{0}^{1}\left(-\frac{\partial \mathcal{H}}{\partial P_{\sigma}^{i}}+y_{\mid i}^{\sigma}\right) \circ \chi d u \\
& =-y^{\sigma} \int_{0}^{1}\left(\frac{\partial \mathcal{H}}{\partial y^{\sigma}} \circ \chi\right) d u-P_{\sigma}^{i} \int_{0}^{1}\left(\frac{\partial \mathcal{H}}{\partial P_{\sigma}^{i}} \circ \chi\right) d u+\frac{1}{2}\left(P_{\sigma}^{i} y_{\mid i}^{\sigma}-y^{\sigma} P_{\sigma \mid i}^{i}\right) \\
& =-\mathcal{H}+P_{\sigma}^{i} y_{\mid i}^{\sigma} \\
& + \text { a term of the form } d_{i} f^{i}, \text { providing zero Euler-Lagrange expressions. }
\end{aligned}
$$

If $D \subset X$ is a connected compact $n$-dimensional submanifold with boundary, the action yielding the Hamilton-De Donder equations is therefore the function

$$
\psi \mapsto \int_{D} J^{1} \psi^{*}\left(-\mathcal{H}+P_{\sigma}^{i} y_{\mid i}^{\sigma}\right) d x^{1} \wedge \cdots \wedge d x^{n}
$$

on the set of sections of the fibred manifold $\tau: J^{*} Y \rightarrow X$ satisfying $D \subset \operatorname{Dom} \psi$ and $\operatorname{Im} \psi \subset \operatorname{Dom} \mathcal{L}_{\mathrm{h}}$; variations are then vector fields on $U \subset J^{*} Y$ projectable to $X$. Note that the Lagrangian $\mathcal{L}_{\mathrm{h}}$ is a first order Lagrangian that is affine in the first derivatives, so that its Euler-Lagrange equations are indeed first order equations.

There is an alternative, more geometric, approach to this problem, obtained from a direct use of the fibred manifold $\tau: J^{*} Y \rightarrow X$ and its jet prolongation $\tau_{1}: J^{1}\left(J^{*} Y\right) \rightarrow X$. 
Let $\mathrm{h}$ and $\mathrm{p}_{1}$ denote the horizontalization and 1-contactization operators with respect to $\tau_{1}$, and let

$$
\vartheta^{\sigma}=d y^{\sigma}-y_{\mid j}^{\sigma} d x^{j}, \quad \pi_{\sigma}^{i}=d P_{\sigma}^{i}-P_{\sigma \mid j}^{i} d x^{j}
$$

denote the corresponding local contact 1-forms. We use the pullback $\Theta_{\mathrm{h}}=-\mathcal{H} \omega_{0}+$ $P_{\sigma}^{i} d y^{\sigma} \wedge \omega_{i}$ of the canonical $n$-form on $J^{*} Y$, defined on the domain $U$ of the hamiltonian section $\mathrm{h}$.

Theorem 2.3. The Hamilton-De Donder equations of the $(n+1)$-form $\Omega_{\mathrm{h}}=d \Theta_{\mathrm{h}}$ are the Euler-Lagrange equations of the Lagrangian

(2.6) $\Lambda_{\mathrm{h}}=\mathrm{h}\left(\Theta_{\mathrm{h}}\right)=\left(-\mathcal{H}+P_{\sigma}^{i} y_{\mid i}^{\sigma}\right) \omega_{0}$.

Proof. Although this follows immediately from the definition of the Tonti Lagrangian

$$
\mathcal{L}_{\mathrm{h}}=-\mathcal{H}+P_{\sigma}^{i} y_{\mid i}^{\sigma}
$$

given above, we can also provide a different argument: it is easy to see that the Cartan form $\Theta_{\Lambda_{\mathrm{h}}}$ of the Lagrangian $\Lambda_{\mathrm{h}}$ is the pullback of $\Theta_{\mathrm{h}}$ to $J^{1}\left(J^{*} Y\right)$, so the Euler-Lagrange equations for $\Lambda_{\mathrm{h}}$ are exactly equations (2.2) and are given in coordinates by (2.3).

For this reason we shall call the Euler-Lagrange form $E_{\mathrm{h}}$ of the Lagrangian $\Lambda_{\mathrm{h}}=$ $\mathrm{h}\left(\Theta_{\mathrm{h}}\right)$ the Hamilton-De Donder form; its coordinate expression is of course

$$
\begin{aligned}
E_{\mathrm{h}} & =\mathrm{p}_{1} d \Theta_{\Lambda_{\mathrm{h}}}=\mathrm{p}_{1} \Omega_{\mathrm{h}}=\mathrm{p}_{1}\left(-d \mathcal{H} \wedge \omega_{0}+\left(\pi_{\sigma}^{i}+P_{\sigma \mid j}^{i} d x^{j}\right) \wedge\left(\vartheta^{\sigma}+y_{\mid j}^{\sigma} d x^{j}\right) \wedge \omega_{i}\right) \\
& =-\left(\frac{\partial \mathcal{H}}{\partial y^{\sigma}}+P_{\sigma \mid i}^{i}\right) \vartheta^{\sigma} \wedge \omega_{0}-\left(\frac{\partial \mathcal{H}}{\partial P_{\sigma}^{i}}-y_{\mid i}^{\sigma}\right) \pi_{\sigma}^{i} \wedge \omega_{0} .
\end{aligned}
$$

As the Hamilton-De Donder equations are variational, we shall use the term HamiltonDe Donder extremal to denote any section $\psi$ of $\tau: J^{*} Y \rightarrow X$ that is a solution of the Hamilton-De Donder equations.

\subsection{The inverse problem}

The Hamilton-De Donder equations are a system of first order PDEs which, as we have seen, come from a variational principle on the dual jet bundle. We may therefore consider the inverse problem which can be stated in general terms as follows.

Given a system of first-order PDEs

$$
E_{J}\left(x^{i}, z^{K}, z_{i}^{K}\right)=0,
$$

is there a Lagrangian such that these equations are equations for the extremals of that Lagrangian?

This is a familiar problem which, when expressed more precisely as a question about the corresponding dynamical form, may be considered on two levels, local and global. In 
both cases the solution is well known. In our case we let $\tau: J^{*} Y \rightarrow X$ be the reduced dual bundle, and we say that a system of $m+m n$ differential equations

$$
\frac{\partial y^{\sigma}}{\partial x^{i}}-F_{i}^{\sigma}=0, \quad \frac{\partial P_{\sigma}^{j}}{\partial x^{j}}+G_{\sigma}=0, \quad 1 \leq \sigma \leq m, 1 \leq i \leq n
$$

for local sections $\psi(x)=\left(x^{i}(x), y^{\sigma}(x), P_{\sigma}^{j}(x)\right)$ of $\tau$ is locally variational if, for each point of $J^{*} Y$, there is a Lagrangian function $L$ defined on some neighbourhood of that point such that the Euler-Lagrange expressions of $L$ coincide with the given equations "as they stand". Applying the known results, we obtain the following.

Corollary 2.4. The system of equations (2.8) is locally variational if and only if the functions $F_{i}^{\sigma}$ and $G_{\sigma}$ obey the integrability conditions

(2.9) $\frac{\partial G_{\sigma}}{\partial y^{\nu}}-\frac{\partial G_{\nu}}{\partial y^{\sigma}}=0, \quad \frac{\partial F_{i}^{\sigma}}{\partial P_{\nu}^{k}}-\frac{\partial F_{k}^{\nu}}{\partial P_{\sigma}^{i}}=0, \quad \frac{\partial G_{\sigma}}{\partial P_{\nu}^{k}}-\frac{\partial F_{k}^{\nu}}{\partial y^{\sigma}}=0$.

If this is the case then, locally,

$$
F_{i}^{\sigma}=\frac{\partial \mathcal{H}}{\partial P_{\sigma}^{i}}, \quad G_{\sigma}=\frac{\partial \mathcal{H}}{\partial y^{\sigma}},
$$

where

$$
\mathcal{H}=y^{\sigma} \int_{0}^{1}\left(G_{\sigma} \circ \chi\right) d u+P_{\sigma}^{j} \int_{0}^{1}\left(F_{j}^{\sigma} \circ \chi\right) d u .
$$

A corresponding local Lagrangian function on an open subset of $J^{1}\left(J^{*} Y\right)$ is

$$
\mathcal{L}=-\mathcal{H}+P_{\sigma}^{i} y_{\mid i}^{\sigma} .
$$

Furthermore, the function $\mathcal{H}$ defines a local Hamiltonian section $\mathrm{h}$ of the projection $\rho$ : $J^{\dagger} Y \rightarrow J^{*} Y$ such that equations (2.8) represent a local Hamiltonian system.

In the language of PDEs this result concerns a system of first order PDEs for a vector function and a tensor function of $n$ independent variables, such that the equations for the vector function are in the explicit form, and those for the tensor function are in divergence form.

\section{Hamiltonian connections}

\subsection{Fields of Hamilton-De Donder extremals}

We now investigate the geometric meaning of the Hamilton-De Donder equations by considering them in the context of Ehresmann connections. We can see immediately, from their structure, that the Hamilton-De Donder equations are not equations for the paths of a single Ehresmann connection when $n \geq 2$, in contrast to the case of Hamiltonian mechanics. We can, however, construct various local Ehresmann connections whose solutions are always Hamilton-De Donder extremals. 
To see this, consider for example the coordinate equations

$$
\frac{\partial \psi^{\sigma}}{\partial x^{i}}=\frac{\partial \mathcal{H}}{\partial P_{\sigma}^{i}}, \quad \frac{\partial \psi_{\sigma}^{1}}{\partial x^{1}}=-\frac{\partial \mathcal{H}}{\partial y^{\sigma}}, \quad \frac{\partial \psi_{\sigma}^{1}}{\partial x^{a}}=0, \quad \frac{\partial \psi_{\sigma}^{b}}{\partial x^{i}}=0
$$

where the indices $a, b$ run from 2 to $n$, so that these are the equations of a connection in the given chart. Any local integral section of this connection will clearly be a HamiltonDe Donder extremal, although the converse need not hold: it need not be the case that every Hamilton-De Donder extremal is also a solution of the connection equations. We can obviously write down many other sets of connection equations satisfying a similar property.

To approach this problem more systematically, let $\hat{\Gamma}$ denote a local Ehresmann connection on $\tau: J^{*} Y \rightarrow X$, so that $\hat{\Gamma}$ is a local section of the fibred manifold $\tau_{1,0}$ : $J^{1}\left(J^{*} Y\right) \rightarrow J^{*} Y$. Suppose that the domain of $\hat{\Gamma}$ contains $U$, the domain of $\Omega_{\mathrm{h}}$, and that $\hat{\Gamma}$ is represented by its horizontal projector

$$
\Gamma=d x^{j} \otimes\left(\frac{\partial}{\partial x^{j}}+\Gamma_{j}^{\sigma} \frac{\partial}{\partial y^{\sigma}}+\Gamma_{\sigma j}^{i} \frac{\partial}{\partial P_{\sigma}^{i}}\right),
$$

where $\Gamma_{j}^{\sigma}=y_{\mid j}^{\sigma} \circ \hat{\Gamma}$ and $\Gamma_{\sigma j}^{i}=P_{\sigma \mid j}^{i} \circ \hat{\Gamma}$ are the components of the section $\hat{\Gamma}$ in the local coordinates; we then see that

$$
i_{\Gamma} \Omega_{\mathrm{h}}=(n-1) \Omega_{\mathrm{h}}-\left(\Gamma_{\sigma i}^{i}+\frac{\partial \mathcal{H}}{\partial y^{\sigma}}\right) d y^{\sigma} \wedge \omega_{0}+\left(\Gamma_{i}^{\sigma}-\frac{\partial \mathcal{H}}{\partial P_{\sigma}^{i}}\right) d P_{\sigma}^{i} \wedge \omega_{0}
$$

If a local section $\psi$ of $\tau: J^{*} Y \rightarrow X$ is an integral section of $\hat{\Gamma}$ then it will satisfy the condition $\hat{\Gamma} \circ \psi=j^{1} \psi$, so that in coordinates it will satisfy

$$
\frac{\partial \psi^{\sigma}}{\partial x^{j}}=\Gamma_{j}^{\sigma}, \quad \frac{\partial \psi_{\sigma}^{i}}{\partial x^{j}}=\Gamma_{\sigma j}^{i} .
$$

We therefore have the following result (cf. [5]).

Theorem 3.1. If an Ehresmann connection $\hat{\Gamma}$ on $\tau: J^{*} Y \rightarrow X$ satisfies the compatibility condition

(3.1) $i_{\Gamma} \Omega_{\mathrm{h}}=(n-1) \Omega_{\mathrm{h}}$

then any integral section of $\hat{\Gamma}$ is a solution of the Hamilton-De Donder equations (2.3), and so is a Hamilton-De Donder extremal.

We can express this compatibility condition in terms of the Hamilton-De Donder form $E_{\mathrm{h}}=\mathrm{p}_{1} \Omega_{\mathrm{h}}$.

Theorem 3.2. For any Ehresmann connection $\hat{\Gamma}$ on the fibred manifold $\tau_{1,0}: J^{1}\left(J^{*} Y\right) \rightarrow$ $J^{*} Y$ the compatibility condition (3.1) is equivalent to the condition

(3.2) $\hat{\Gamma}^{*} E_{\mathrm{h}}=0$. 
Proof. By (2.7),

$$
\hat{\Gamma}^{*} E_{\mathrm{h}}=-\left(\frac{\partial \mathcal{H}}{\partial y^{\sigma}}+\Gamma_{\sigma i}^{i}\right) \vartheta^{\sigma} \wedge \omega_{0}-\left(\frac{\partial \mathcal{H}}{\partial P_{\sigma}^{i}}-\Gamma_{i}^{\sigma}\right) \pi_{\sigma}^{i} \wedge \omega_{0},
$$

so that $\hat{\Gamma}^{*} E_{\mathrm{h}}=0$ if and only if

$$
\Gamma_{i}^{\sigma}=\frac{\partial \mathcal{H}}{\partial P_{\sigma}^{i}}, \quad \Gamma_{\sigma i}^{i}=-\frac{\partial \mathcal{H}}{\partial y^{\sigma}},
$$

and this holds if and only if equation (3.1) holds.

We shall therefore call connections compatible with a Hamilton-De Donder system fields of Hamilton-De Donder extremals. Note, however, that in general there is no guarantee that such a connection will have any integral sections at all, even if the Hamilton-De Donder equations have solutions.

Although connections compatible with a Hamilton-De Donder system are not unique, we may nevertheless describe them all locally.

Theorem 3.3. Let $\Omega_{\mathrm{h}}$ be a Hamilton-De Donder system on $U \subset J^{*} Y$. If $\hat{\Gamma}$ is an Ehresmann connection on $\tau: J^{*} Y \rightarrow X$ that is compatible with $\Omega_{\mathrm{h}}$ then $\hat{\Gamma}$ is described locally by the horizontal projector

$$
\Gamma=d x^{j} \otimes\left(\frac{\partial}{\partial x^{j}}+\frac{\partial \mathcal{H}}{\partial P_{\sigma}^{j}} \frac{\partial}{\partial y^{\sigma}}-\left(\frac{1}{n} \delta_{j}^{i} \frac{\partial \mathcal{H}}{\partial y^{\sigma}}+F_{\sigma j}^{i}\right) \frac{\partial}{\partial P_{\sigma}^{i}}\right)
$$

where for each index $\sigma$, the matrix of functions $\left(F_{\sigma j}^{i}\right)$ is an arbitrary $(n \times n)$-matrix on $U$, traceless at each point of $U$.

Proof. It is sufficient to check the formula for $\Gamma_{\sigma j}^{i}$. Fixing $\sigma$, considering $\left(\Gamma_{\sigma j}^{i}\right)$ as a $(n \times n)$-matrix on $U$ and applying the trace decomposition formula, we see that $\Gamma_{\sigma j}^{i}$ may be expressed as

(3.4) $\Gamma_{\sigma j}^{i}=\left(\Gamma_{\sigma j}^{i}-\frac{1}{n} \delta_{j}^{i} \operatorname{Tr} \Gamma_{\sigma}\right)+\frac{1}{n} \delta_{j}^{i} \operatorname{Tr} \Gamma_{\sigma}$.

Putting

$$
-F_{\sigma j}^{i}=\Gamma_{\sigma j}^{i}-\frac{1}{n} \delta_{j}^{i} \operatorname{Tr} \Gamma_{\sigma}
$$

and using the fact that $\operatorname{Tr} \Gamma_{\sigma}=-\partial H / \partial y^{\sigma}$, we obtain the result.

So we have found a family of Ehresmann connections such that every integral section of each connection is a Hamilton-De Donder extremal. In particular, for every integrable connection $\hat{\Gamma}$ in this family (in the sense of Frobenius complete integrability) this means that the corresponding maximal Hamilton-De Donder extremals form a $n$-dimensional foliation of the open submanifold $\operatorname{Dom} \hat{\Gamma} \subset J^{*} Y$.

It is now natural to ask whether the family of Ehresmann connections characterized by Theorem 3.3 includes (at least locally) all Hamilton-De Donder extremals. The answer is positive: every Hamilton-De Donder extremal is locally an integral section of some compatible connection, which is maximal in the sense that it is defined on the domain $U$ of $\mathrm{h}$. 
Lemma 3.4. If $\mathrm{h}$ is a section of $\rho: J^{\dagger} Y \rightarrow J^{*} Y$ defined on $U \subset J^{*} Y$ then there is a connection $\hat{\Gamma}_{0}$ on $U$ satisfying $i_{\Gamma_{0}} \Omega_{\mathrm{h}}=(n-1) \Omega_{\mathrm{h}}$. If, in particular, $\mathrm{h}$ is a global section of $\rho: J^{\dagger} Y \rightarrow J^{*} Y$ then $\hat{\Gamma}_{0}$ is a global connection.

Proof. We consider the global case first, and use a partition of unity argument. Let $\left(V_{\alpha}\right)$ be an open cover of $Y$ by domains of adapted charts and put $V_{\alpha}^{*}=\left(\pi_{1,0}^{*}\right)^{-1}\left(V_{\alpha}\right)$, so that $\left(V_{\alpha}^{*}\right)$ is an open cover of $J^{*} Y$ by domains of adapted charts. Let $\left(f_{\alpha}\right)$ be a partition of unity subordinate to $\left(V_{\alpha}^{*}\right)$.

In the chart corresponding to $V_{\alpha}^{*}$ define the functions $\Gamma_{i}^{\sigma}$ and $\Gamma_{\sigma j}^{i}$ by

$$
\Gamma_{i}^{\sigma}=\frac{\partial \mathcal{H}}{\partial P_{\sigma}^{i}}, \quad \Gamma_{\sigma j}^{i}=-\frac{1}{n} \delta_{j}^{i} \frac{\partial \mathcal{H}}{\partial y^{\sigma}} ;
$$

if we put

$$
\Gamma_{\alpha}=d x^{j} \otimes\left(\frac{\partial}{\partial x^{j}}+\Gamma_{j}^{\sigma} \frac{\partial}{\partial y^{\sigma}}+\Gamma_{\sigma j}^{i} \frac{\partial}{\partial P_{\sigma}^{i}}\right)
$$

then $\Gamma_{\alpha}$ is the horizontal projector of a connection defined on $V_{\alpha}^{*}$ satisfying the condition that $i_{\Gamma_{\alpha}} \Omega_{\mathrm{h}}=(n-1) \Omega_{\mathrm{h}}$.

Now put $\Gamma_{0}=\sum_{\alpha} f_{\alpha} \Gamma_{\alpha}$, so that

$$
i_{\Gamma_{0}} \Omega_{\mathrm{h}}=\sum_{\alpha} i_{f_{\alpha} \Gamma_{\alpha}} \Omega_{\mathrm{h}}=\sum_{\alpha} f_{\alpha}\left(i_{\Gamma_{\alpha}} \Omega_{\mathrm{h}}\right)=\left(\sum_{\alpha} f_{\alpha}\right)(n-1) \Omega_{\mathrm{h}}=(n-1) \Omega_{\mathrm{h}} .
$$

The argument when $\mathrm{h}$ is defined on a nonempty open subset of $J^{*} Y$ is identical.

Theorem 3.5. Let $\mathrm{h}$ be a section of $\rho: J^{\dagger} Y \rightarrow J^{*} Y$ defined on $U \subset J^{*} Y$, and suppose that $W$ is a nonempty open subset of $\tau(U) \subset X$. Let $\psi$ be a local section of $\tau: J^{*} Y \rightarrow X$ defined on $W$ and satisfying $\psi^{*}\left(i_{\xi} \Omega_{\mathrm{h}}\right)=0$ for every vertical vector field $\xi$ on $\tau^{-1}(W)$. For each $x \in W$ there is then a connection $\hat{\Gamma}$, defined on $U \subset J^{*} Y$ and satisfying the compatibility condition $i_{\Gamma} \Omega_{\mathrm{h}}=(n-1) \Omega_{\mathrm{h}}$, such that for some neighbourhood $N$ of $\psi(x)$ the restriction $\left.\psi\right|_{\tau(N)}$ is an integral section of $\hat{\Gamma}$.

Proof. Let $V$ be the domain of a chart on $Y$ with $\pi_{1,0}^{*}(\psi(x)) \in V$, and put $\bar{V}=$ $V \cap \pi^{-1}(W)$ and $\bar{W}=\pi(\bar{V})$. Put $\bar{V}^{*}=\left(\pi_{1,0}^{*}\right)^{-1}(\bar{V})$, and let $\nu: \bar{N} \rightarrow \psi(\bar{W})$ be an open tubular neighbourhood of the image submanifold $\psi(\bar{W}) \subset \bar{V}^{*}$. Define the functions $\Gamma_{i}^{\sigma}$ and $\Gamma_{\sigma j}^{i}$ on $\bar{N}$ by

$$
\Gamma_{i}^{\sigma}=\frac{\partial \mathcal{H}}{\partial P_{\sigma}^{i}}, \quad \Gamma_{\sigma j}^{i}=\nu^{*}\left(\frac{\partial \psi_{\sigma}^{i}}{\partial x^{j}}\right)-\frac{1}{n} \delta_{j}^{i}\left(\nu^{*}\left(\frac{\partial \psi_{\sigma}^{k}}{\partial x^{k}}\right)+\frac{\partial \mathcal{H}}{\partial y^{\sigma}}\right),
$$

so putting

$$
\bar{\Gamma}=d x^{j} \otimes\left(\frac{\partial}{\partial x^{j}}+\Gamma_{j}^{\sigma} \frac{\partial}{\partial y^{\sigma}}+\Gamma_{\sigma j}^{i} \frac{\partial}{\partial P_{\sigma}^{i}}\right)
$$

we see that $\bar{\Gamma}$ is the horizontal projector of a connection defined on $\bar{N}$ satisfying the conditions that $i_{\bar{\Gamma}} \Omega_{\mathrm{h}}=(n-1) \Omega_{\mathrm{h}}$, and that $\left.\psi\right|_{\tau(\bar{N})}$ is an integral section of $\bar{\Gamma}$. 
Now let $\hat{\Gamma}_{0}$ be a global compatible connection defined on $U \subset J^{*} Y$ as in Lemma 3.4. Let $(f, g)$ be a partition of unity subordinate to the open cover $(\bar{N}, U-\{\psi(x)\})$ of $U$, and let $N \subset \bar{N}$ be a neighbourhood of $\psi(x)$ such that $\left.f\right|_{N}=1$ and $\left.g\right|_{N}=0$. Then $\Gamma=f \bar{\Gamma}+g \Gamma_{0}$ is the horizontal projector of a connection on $U \subset J^{*} Y$ satisfying the compatibility condition $i_{\Gamma} \Omega_{\mathrm{h}}=(n-1) \Omega_{\mathrm{h}}$, and such that the restriction $\left.\psi\right|_{\tau(N)}$ is an integral section of $\hat{\Gamma}$.

Combining Theorems 3.1 and 3.5 we obtain the following "Main Theorem".

Theorem 3.6. Every solution of the Hamilton-De Donder equations can be locally embedded in a field of Hamilton-De Donder extremals.

\subsection{Integrable Hamiltonian connections}

Let $\hat{\Gamma}$ be an Ehresmann connection on $U \subset J^{*} Y$, given by its horizontal projector

$$
\Gamma=d x^{j} \otimes\left(\frac{\partial}{\partial x^{j}}+\Gamma_{j}^{\sigma} \frac{\partial}{\partial y^{\sigma}}+\Gamma_{\sigma j}^{i} \frac{\partial}{\partial P_{\sigma}^{i}}\right),
$$

and let

$$
\Gamma_{(j)}=\frac{\partial}{\partial x^{j}}+\Gamma_{j}^{\sigma} \frac{\partial}{\partial y^{\sigma}}+\Gamma_{\sigma j}^{i} \frac{\partial}{\partial P_{\sigma}^{i}}
$$

be local generators of the corresponding horizontal distribution. The connection is flat, in other words its curvature vanishes, if and only if the horizontal distribution is Frobenius integrable. Notice that the Lie bracket of any two horizontal vector fields (3.5) is a vector field vertical over $X$, so that the integrability conditions are

$$
\left[\Gamma_{(i)}, \Gamma_{(j)}\right]=0,
$$

or, more explicitly,

$$
\Gamma_{(i)}\left(\Gamma_{j}^{\sigma}\right)-\Gamma_{(j)}\left(\Gamma_{i}^{\sigma}\right)=0, \quad \Gamma_{(i)}\left(\Gamma_{\sigma j}^{k}\right)-\Gamma_{(j)}\left(\Gamma_{\sigma i}^{k}\right)=0 .
$$

Assuming that $\hat{\Gamma}$ is compatible with $\Omega_{\mathrm{h}}$, these integrability conditions take the form

$$
\begin{aligned}
& \Gamma_{(i)}\left(\frac{\partial \mathcal{H}}{\partial P_{\sigma}^{j}}\right)-\Gamma_{(j)}\left(\frac{\partial \mathcal{H}}{\partial P_{\sigma}^{i}}\right)=0, \\
& \Gamma_{(i)}\left(\frac{1}{n} \delta_{j}^{k} \frac{\partial \mathcal{H}}{\partial y^{\sigma}}+F_{\sigma j}^{k}\right)-\Gamma_{(j)}\left(\frac{1}{n} \delta_{i}^{k} \frac{\partial \mathcal{H}}{\partial y^{\sigma}}+F_{\sigma i}^{k}\right)=0 .
\end{aligned}
$$

Rewriting them as conditions for the unknown functions $F_{\sigma j}^{i}$ we can see that the first set represents algebraic conditions on the functions $F_{\sigma j}^{i}$, while the second represents differential conditions on these functions. We therefore obtain the following result.

Proposition 3.7. Let $\hat{\Gamma}$ be an Ehresmann connection on $U \subset J^{*} Y$ compatible with the Hamilton-De Donder form $\Omega_{\mathrm{h}}$; then $\hat{\Gamma}$ is flat if and only if the horizontal distribution $\operatorname{span}\left\{\Gamma_{(i)}, 1 \leq i \leq n\right\}$ is completely integrable, and this holds if and only if the functions $F_{\sigma i}^{j}$ in the coordinate expression (3.3) satisfy 
(1) for each index $\sigma, \operatorname{Tr}\left(F_{\sigma}\right)=0$;

(2) algebraic conditions

$$
\begin{aligned}
i \neq j: \quad F_{\sigma i}^{k} \frac{\partial^{2} \mathcal{H}}{\partial P_{\nu}^{j} \partial P_{\sigma}^{k}} & -F_{\sigma j}^{k} \frac{\partial^{2} \mathcal{H}}{\partial P_{\nu}^{i} \partial P_{\sigma}^{k}}=\frac{1}{n} \frac{\partial \mathcal{H}}{\partial y^{\sigma}}\left(\frac{\partial^{2} \mathcal{H}}{\partial P_{\nu}^{j} \partial P_{\sigma}^{i}}-\frac{\partial^{2} \mathcal{H}}{\partial P_{\nu}^{i} \partial P_{\sigma}^{j}}\right) \\
& +\frac{\partial^{2} \mathcal{H}}{\partial x^{j} \partial P_{\nu}^{i}}-\frac{\partial^{2} \mathcal{H}}{\partial x^{i} \partial P_{\nu}^{j}}+\frac{\partial \mathcal{H}}{\partial P_{\sigma}^{j}} \frac{\partial^{2} \mathcal{H}}{\partial y^{\sigma} \partial P_{\nu}^{i}}-\frac{\partial \mathcal{H}}{\partial P_{\sigma}^{i}} \frac{\partial^{2} \mathcal{H}}{\partial y^{\sigma} \partial P_{\nu}^{j}}
\end{aligned}
$$

(3) differential conditions

$$
\begin{array}{ll}
i \neq j=k: & \Gamma_{(i)}\left(F_{\sigma j}^{j}\right)-\Gamma_{(j)}\left(F_{\sigma i}^{j}\right)=-\frac{1}{n} \Gamma_{(i)}\left(\frac{\partial \mathcal{H}}{\partial y^{\sigma}}\right) \quad \text { no summation } \\
i \neq j \neq k: & \Gamma_{(i)}\left(F_{\sigma j}^{k}\right)-\Gamma_{(j)}\left(F_{\sigma i}^{k}\right)=0 .
\end{array}
$$

Corollary 3.8. If $\hat{\Gamma}$ is a flat connection on $U$ compatible with $\Omega_{\mathrm{h}}$ then the integral sections of $\hat{\Gamma}$ form an $n$-dimensional foliation of $U$, and the leaves of this foliation are solutions of the Hamilton-De Donder equations. It follows that the Cauchy problem for the given Hamilton-De Donder equations has at least one maximal solution for any given initial condition on $U$, corresponding to the unique maximal integral manifold passing through that point of $U$.

Definition 3.9. Let $U$ be an open subset of $J^{*} Y$. We say that a Hamilton-De Donder system is Cauchy integrable on $U$ if the Cauchy problem for the given Hamilton-De Donder equations has, for every initial condition in $U$, at least one maximal solution.

We therefore see that a sufficient condition for the Cauchy integrability of $\Omega_{\mathrm{h}}$ on $U$ is the existence on $U$ of a flat Ehresmann connection compatible with $\Omega_{\mathrm{h}}$. Indeed, there is a distinguished family of Hamiltonian systems with the following desirable integrability properties.

Definition 3.10. We say that a Hamilton-De Donder system $\Omega_{\mathrm{h}}$ is completely integrable if every solution (that is, every local section $\psi$ of $J^{*} Y$ satisfying the Hamilton-De Donder equations) can be locally embedded in a flat compatible Ehresmann connection.

\section{Hamilton-De Donder equations in $J^{1} Y$}

\subsection{The Goldschmidt-Sternberg setting}

So far we have considered Hamiltonian systems in dual jet bundles, and the corresponding Hamilton-De Donder equations have arisen from the canonical multisymplectic structure as equations for sections of the bundle $\tau: J^{*} Y \rightarrow X$, dual to a jet bundle $\pi_{1}: J^{1} Y \rightarrow$ $X$. This model generalizes Hamiltonian systems on symplectic manifolds to the case of several independent variables.

We shall now take a different approach, looking at Hamilton-De Donder theory in the setting of Goldschmidt and Sternberg [8], who considered Hamilton's equations as 
equations for sections of the bundle $\pi_{1}: J^{1} Y \rightarrow X$ that arise from a variational problem on $\pi: Y \rightarrow X$.

Let $\lambda$ be a Lagrangian on $J^{1} Y$, and consider its Poincaré-Cartan form

$$
\theta_{\lambda}=L \omega_{0}+\frac{\partial L}{\partial y_{i}^{\sigma}} \omega^{\sigma} \wedge \omega_{i}
$$

If the Poincaré-Cartan form is expressed in the canonical basis given by differentials of the coordinate functions, rather than in the basis adapted to the contact structure as above, the components of $\theta_{\lambda}$ are called the momenta and the Hamiltonian of $\lambda$. We then obtain

(4.2) $\theta_{\lambda}=-H \omega_{0}+p_{\sigma}^{i} d y^{\sigma} \wedge \omega_{i}$

where the momenta and Hamiltonian are local functions on $J^{1} Y$ given by

$$
p_{\sigma}^{i}=\frac{\partial L}{\partial y_{i}^{\sigma}}, \quad H=-L+\frac{\partial L}{\partial y_{i}^{\sigma}} y_{i}^{\sigma}=-L+p_{\sigma}^{i} y_{i}^{\sigma}
$$

Using the first variation formula it can be shown that a local section $\gamma$ of $\pi$ is an extremal of the action of $\lambda$ if and only if it satisfies the Euler-Lagrange equations

(4.4) $j^{1} \gamma^{*} i_{\xi} d \theta_{\lambda}=0$ for every vector field $\xi$ on $J^{1} Y$ vertical over $X$.

Denote by $\mathcal{D}_{\lambda}$ the exterior differential system generated by $n$-foms $i_{\xi} d \theta_{\lambda}$ where $\xi$ runs over all vector fields on $J^{1} Y$ that are vertical over $X$. Then, in terms of exterior differential systems theory, equations (4.4) can be viewed as equations for special solutions of $\mathcal{D}_{\lambda}$, namely those which are local sections of $\pi_{1}$ and have the form of prolongations of local sections of $\pi$. The equations for all integral sections $\phi$ of $\mathcal{D}_{\lambda}$ (that is, sections that need not be prolongations) take the form

(4.5) $\phi^{*} i_{\xi} d \theta_{\lambda}=0$ for every vertical vector field $\xi$ on $J^{1} Y$,

and are called Hamilton-De Donder equations: the terminology arises from the local expression of these equations in the Legendre coordinates of a regular Lagrangian. Recall that a Lagrangian is called regular if

(4.6) $\operatorname{det}\left(\frac{\partial^{2} L}{\partial y_{i}^{\sigma} \partial y_{j}^{\nu}}\right) \neq 0$

the momenta $p_{\sigma}^{i}$ of a regular Lagrangian are independent, and can be used to define new local coordinates $\left(x^{i}, y^{\sigma}, p_{\sigma}^{i}\right)$ on $J^{1} Y$ called Legendre coordinates. In these Legendre coordinates, and using the Hamiltonian $H$, we see that local generators of $\mathcal{D}_{\lambda}$ are

(4.7) $-\frac{\partial H}{\partial y^{\sigma}} \omega_{0}-d p_{\sigma}^{i} \wedge \omega_{i}, \quad-\frac{\partial H}{\partial p_{\sigma}^{i}} \omega_{0}+d y^{\sigma} \wedge \omega_{i}$. 
We may then use equations (4.5) to obtain

(4.8) $\frac{\partial y^{\sigma}}{\partial x^{i}}=\frac{\partial H}{\partial p_{\sigma}^{i}}, \quad \frac{\partial p_{\sigma}^{i}}{\partial x^{i}}=-\frac{\partial H}{\partial y^{\sigma}}$

the Hamilton equations as introduced by De Donder [4].

We can see that equations (4.5) are more general than (4.8), as they are coordinateindependent and do not require regularity of the Lagrangian. Compared with the EulerLagrange equations, equations (4.5) generally have more solutions. It can, however, be shown that if $\lambda$ satisfies the above regularity condition then the Hamilton-De Donder equations (4.5) are equivalent to the Euler-Lagrange equations, in the sense that solutions of the former equations must now necessarily be prolongations, and so are in bijective correspondence with solutions of the latter equations.

Following Krupka and Stepankova [12], we may now consider the first jet prolongation of the fibred manifold $\pi_{1}$. Given a Lagrangian $\lambda$ on $J^{1} Y$, put

$$
\tilde{\lambda}=\tilde{h} \theta_{\lambda}
$$

where $\tilde{h}$ represents horizontalization with respect to the projection $\pi_{1}$, so that $\tilde{\lambda}$ is a horizontal form (a Lagrangian) on $J^{1}\left(J^{1} Y\right)$. Using $\theta_{\tilde{\lambda}}=\left(\pi_{1}\right)_{1,0}^{*} \theta_{\lambda}$, we see that the Euler-Lagrange form of $\tilde{\lambda}$ is $E_{\tilde{\lambda}}=\tilde{p}_{1} d \theta_{\lambda}$, and that $\left(\pi_{1}\right)_{1,0}^{*} d \theta_{\lambda}$ is projectable to $J^{1} Y$. We immediately obtain the following result [12].

Theorem 4.1. The Hamilton-De Donder equations (4.5) are variational, and they are Euler-Lagrange equations of the Lagrangian $\tilde{\lambda}$.

\subsection{Semispray connections in Hamilton-De Donder theory}

Now consider the bundle of semiholonomic jets $\hat{J}^{2} Y \rightarrow X$, and let $\iota: J^{2} Y \rightarrow \hat{J}^{2} Y$ be the natural inclusion. Let $\hat{\Gamma}$ be a local semiholonomic semispray connection on $J^{1} Y$, so that $\hat{\Gamma}$ is a local section $J^{1} Y \rightarrow \hat{J}^{2} Y$. With such connection we can associate a vector-valued one-form

$$
\Gamma=d x^{i} \otimes\left(\frac{\partial}{\partial x^{i}}+y_{i}^{\sigma} \frac{\partial}{\partial y^{\sigma}}+\Gamma_{i j}^{\sigma} \frac{\partial}{\partial y_{j}^{\sigma}}\right)
$$

called its horizontal form, and also a horizontal distribution spanned locally by the semisprays

$$
\Gamma_{(i)}=\frac{\partial}{\partial x^{i}}+y_{i}^{\sigma} \frac{\partial}{\partial y^{\sigma}}+\Gamma_{i j}^{\sigma} \frac{\partial}{\partial y_{j}^{\sigma}},
$$

where $\Gamma_{i j}^{\sigma}$ are the components of $\hat{\Gamma}$. Using the fibre product decomposition

$$
\hat{J}^{2} Y \cong J^{2} Y \times_{J^{1} Y}\left(\bigwedge^{2} T^{*} X \otimes V Y\right)
$$


([15], Theorem 5.3.4) we see that the symmetric part of a semiholonomic connection $\hat{\Gamma}$ is a holonomic connection $\hat{\Gamma}_{0}$, with $y_{i j}^{\sigma} \circ \hat{\Gamma}_{0}=\Gamma_{(i j)}^{\sigma}$, and the skew-symmetric part is a vector-valued 2 -form on $J^{1} Y$, the torsion

$$
\mathbf{T}=\Gamma_{[i j]}^{\sigma}\left(d x^{i} \wedge d x^{j}\right) \otimes \frac{\partial}{\partial y^{\sigma}}
$$

where for each index $\sigma$ we let $\Gamma_{(i j)}^{\sigma}$ and $\Gamma_{[i j]}^{\sigma}$ denote the symmetric and skew-symmetric parts of the matrix $\Gamma^{\sigma}=\left(\Gamma_{i j}^{\sigma}\right)$. Using the vertical lift correspondence

$$
S:\left(d x^{i} \otimes \frac{\partial}{\partial y^{\sigma}}\right) \mapsto \frac{\partial}{\partial y_{i}^{\sigma}}, \quad S\left(\left(d x^{i} \wedge d x^{j}\right) \otimes \frac{\partial}{\partial y^{\sigma}}\right)=\frac{1}{2}\left(d x^{i} \otimes \frac{\partial}{\partial y_{j}^{\sigma}}-d x^{j} \otimes \frac{\partial}{\partial y_{i}^{\sigma}}\right)
$$

we may therefore write the horizontal projector of $\hat{\Gamma}$ as a sum

$$
\Gamma=d x^{i} \otimes\left(\frac{\partial}{\partial x^{i}}+y_{i}^{\sigma} \frac{\partial}{\partial y^{\sigma}}+\Gamma_{(i j)}^{\sigma} \frac{\partial}{\partial y_{j}^{\sigma}}\right)+\Gamma_{[i j]}^{\sigma} S\left(\left(d x^{i} \wedge d x^{j}\right) \otimes \frac{\partial}{\partial y^{\sigma}}\right) .
$$

A local section $\gamma$ of $\pi$ such that $\operatorname{Im} j^{1} \gamma \subset$ Dom $\hat{\Gamma}$ will be called an integral section of the semiholonomic connection $\hat{\Gamma}$ if

$$
\hat{\Gamma}_{0} \circ j^{1} \gamma=j^{2} \gamma,
$$

and we see that such integral sections are solutions of a system of second order PDEs given by

$$
\frac{\partial^{2} \gamma^{\sigma}}{\partial x^{i} \partial x^{j}}=\Gamma_{(i j)}^{\sigma}
$$

Indeed, if $\phi$ is a local section of $\pi_{1}: J^{1} Y \rightarrow X$ such that $\operatorname{Im} \phi \subset \operatorname{Dom} \hat{\Gamma}$, and if $\hat{\Gamma} \circ \phi=$ $j^{1} \phi \in \hat{J}^{2} Y \subset J^{1} J^{1} Y$ (so that $\phi$ is an integral section in the usual sense of $\hat{\Gamma}$, where the latter is regarded as an ordinary Ehresmann connection on $J^{1} Y \rightarrow X$ ) then

$$
\phi_{i}^{\sigma}=\frac{\partial \phi^{\sigma}}{\partial x^{i}}
$$

so that $\phi=j^{1} \gamma$ for some local section $\gamma$ of $\pi$, and then $\gamma$ is an integral section of $\hat{\Gamma}$ as defined above.

Now given a Lagrangian $\lambda$ on $J^{1} Y$, we say that a (holonomic or semiholonmic) semispray connection $\hat{\Gamma}$ is $\lambda$-compatible if

$$
i_{\Gamma} d \theta_{\lambda}=(n-1) d \theta_{\lambda} .
$$

\section{Theorem 4.2.}

(1) A holonomic semispray connection $\hat{\Gamma}$ is $\lambda$-compatible if and only if $\hat{\Gamma}^{*} E_{\lambda}=0$. 
(2) If the matrix

$$
B_{\sigma \nu}^{i j}=\frac{\partial^{2} L}{\partial y_{i}^{\sigma} \partial y_{j}^{\nu}}
$$

is symmetric in $i, j$, namely

$$
B_{\sigma \nu}^{i j}=\frac{\partial E_{\sigma}}{\partial y_{i j}^{\nu}},
$$

then a semiholonomic semispray connection $\hat{\Gamma}$ is $\lambda$-compatible if and only if $\hat{\Gamma}_{0}^{*} E_{\lambda}=$ 0 .

Proof. The result follows from a direct computation. In fibred coordinates we have

$$
d \theta_{\lambda}=A_{\sigma} \omega^{\sigma} \wedge \omega_{0}+\frac{\partial^{2} L}{\partial y^{\nu} \partial y_{i}^{\sigma}} \omega^{\nu} \wedge \omega^{\sigma} \wedge \omega_{i}+B_{\sigma \nu}^{i j} d y_{j}^{\nu} \wedge \omega^{\sigma} \wedge \omega_{i}
$$

where we have put

$$
A_{\sigma}=\frac{\partial L}{\partial y^{\sigma}}-\frac{\partial^{2} L}{\partial x^{j} \partial y_{j}^{\sigma}}-\frac{\partial^{2} L}{\partial y^{\nu} \partial y_{j}^{\sigma}} y_{j}^{\nu}, \quad B_{\sigma \nu}^{i j}=\frac{\partial^{2} L}{\partial y_{i}^{\sigma} \partial y_{j}^{\nu}}
$$

so that $E_{\sigma}=A_{\sigma}-B_{\sigma \nu}^{i j} y_{i j}^{\nu}=A_{\sigma}-B_{\sigma \nu}^{(i j)} y_{i j}^{\nu}$. We then see that

$$
i_{\Gamma} d \theta_{\lambda}=(n-1) d \theta_{\lambda}+\left(A_{\sigma}-B_{\sigma \nu}^{i j} \Gamma_{i j}^{\nu}\right) \omega^{\sigma} \wedge \omega_{0}=(n-1) d \theta_{\lambda}+\hat{\Gamma}_{0}^{*} E_{\lambda}-B_{\sigma \nu}^{[i j]} \Gamma_{[i j]}^{\nu} \omega^{\sigma} \wedge \omega_{0} .
$$

From now on we shall assume that $\lambda$ is a regular Lagrangian. Recall that in this case

(i) around every point in $J^{1} Y$ there are Legendre coordinates $\left(x^{i}, y^{\sigma}, p_{\sigma}^{j}\right)$;

(ii) the Hamilton-De Donder equations are equivalent to the Euler-Lagrange equations of $\lambda$, so that the only solutions of the Hamilton-De Donder equations are prolongations of extremals.

In Legendre coordinates the horizontal form $\Gamma$ of a semispray connection $\hat{\Gamma}$ takes the form

$$
\Gamma=d x^{i} \otimes\left(\frac{\partial}{\partial x^{i}}+y_{i}^{\sigma} \frac{\partial}{\partial y^{\sigma}}+\Gamma_{\sigma i}^{j} \frac{\partial}{\partial p_{\sigma}^{j}}\right),
$$

where now the $y_{i}^{\sigma}$ are considered as functions of the Legendre coordinates; the transformation rule between the remaining components in fibered and Legendre coordinates becomes

$$
\Gamma_{\sigma i}^{j}=\frac{\partial p_{\sigma}^{j}}{\partial y_{k}^{\nu}} \Gamma_{i k}^{\nu}
$$


In Legendre coordinates we have

$$
d \theta_{\lambda}=-d H \wedge \omega_{0}+d p_{\sigma}^{i} \wedge d y^{\sigma} \wedge \omega_{i} \quad \text { and } \quad y_{i}^{\sigma}=\frac{\partial H}{\partial p_{\sigma}^{i}},
$$

and so the compatibility condition applied to $d \theta_{\lambda}$ gives

$$
\Gamma_{\sigma i}^{i}=-\frac{\partial H}{\partial y^{\sigma}}=\frac{\partial L}{\partial y^{\sigma}} .
$$

Using the results of the previous section, we can now obtain similar results for semispray connections compatible with regular Lagrangians. First we discuss the existence of $\lambda$-compatible connections. From the formulas above it is clear that the local existence is guaranteed: indeed we can obtain such a local connection simply by putting, in any Legendre chart,

$$
\Gamma_{\sigma j}^{i}=-\frac{1}{n} \delta_{j}^{i} \frac{\partial H}{\partial y^{\sigma}} .
$$

But the same partition of unity arguments we used in the proof of Lemma 3.4 can also be applied in the present situation, to give the following result.

Theorem 4.3. Given a regular Lagrangian $\lambda$ on $J^{1} Y$, there exists a $\lambda$-compatible semispray connection $\hat{\Gamma}: J^{1} Y \rightarrow \hat{J}^{2} Y$.

The existence of a family of $\lambda$-compatible connections is again obtained with help of the trace decomposition.

Theorem 4.4. If $\lambda$ is a regular Lagrangian then any local $\lambda$-compatible semispray connection is, in Legendre coordinates, described by the horizontal projector

(4.9) $\Gamma=d x^{j} \otimes\left(\frac{\partial}{\partial x^{j}}+\frac{\partial H}{\partial p_{\sigma}^{j}} \frac{\partial}{\partial y^{\sigma}}-\left(\frac{1}{n} \delta_{j}^{i} \frac{\partial H}{\partial y^{\sigma}}+F_{\sigma j}^{i}\right) \frac{\partial}{\partial p_{\sigma}^{i}}\right)$,

where for each index $\sigma$ the matrix of functions $\left(F_{\sigma j}^{i}\right)$ is a traceless $(n \times n)$-matrix.

Our final task is to establish a relationship between solutions of the compatible connections and solutions of the Euler-Lagrange equations. However, guided by the corresponding theorems in the previous section, and under our regularity assumption on the Lagrangian, the results follow immediately.

Theorem 4.5. Let $\lambda$ be a regular Lagrangian. If a local semispray connection $\hat{\Gamma}: J^{1} Y \rightarrow$ $\hat{J}^{2} Y$ is $\lambda$-compatible then the prolongation of any integral section of $\hat{\Gamma}$ is a solution of the Hamilton-De Donder equations (4.8), so that any integral section is an extremal.

Theorem 4.6. Let $\lambda$ be a regular Lagrangian on $J^{1} Y$, and let $\gamma$ be a local section of $\pi: Y \rightarrow X$ defined on a nonempty open subset $W \subset X$ and satisfying the Euler-Lagrange equations $j^{1} \gamma^{*} i_{\xi} d \theta_{\lambda}=0$ for every vertical vector field $\xi$ on $\pi_{1}^{-1}(W)$. For each $x \in W$ there is a $\lambda$-compatible semispray connection $\hat{\Gamma}$ such that, for some neighbourhood $N$ of $j^{1} \gamma(x)$, the restriction $\left.\gamma\right|_{\pi_{1}(N)}$ is an integral section of $\hat{\Gamma}$. 
Once again the proof is completely analogous to the proof of Theorem 3.5, and relies on the construction of a suitable compatible semispray connection defined on an open tubular neighborhood $\nu$ around the image of the given prolonged extremal. If we denote by $\left(x^{i}, u^{\sigma}, v_{\sigma}^{j}\right)$ the components of $j^{1} \gamma$ in Legendre coordinates, we can take the "momentum components" of $\hat{\Gamma}$ in the form

$$
\Gamma_{\sigma j}^{i}=\nu^{*}\left(\frac{\partial v_{\sigma}^{i}}{\partial x^{j}}\right)-\frac{1}{n} \delta_{j}^{i}\left(\nu^{*}\left(\frac{\partial v_{\sigma}^{k}}{\partial x^{k}}\right)+\frac{\partial H}{\partial y^{\sigma}}\right)
$$

A global compatible semispray connection such that $\gamma$ is its (local) integral section is then obtained by the partition of unity arguments from the local connection above and a global connection, the existence of which we have proved in Theorem 4.3.

In summary, therefore, for every regular Lagrangian we have found a family of semispray connections, parametrized by traceless matrices, and such that every integral section of each connection is an extremal, a solution of the Euler-Lagrange equations. Moreover, the integral sections of this family of semispray connections include, at least locally, all extremals.

Remark 4.7. If we consider the integrability of $\lambda$-compatible holonomic semispray connections, we have symmetry of the connection components in fibered coordinates, and we know that $y_{i}^{\sigma}=\partial H / \partial p_{i}^{\sigma}$. Consequently, the integrability conditions for the connection are simpler. The "algebraic conditions" for the unknown functions $F$

$$
\Gamma_{(i)}\left(\frac{\partial H}{\partial p_{\sigma}^{j}}\right)=\Gamma_{(j)}\left(\frac{\partial H}{\partial p_{\sigma}^{i}}\right)
$$

become just

$$
\Gamma_{(i)}\left(y_{j}^{\sigma}\right)=\Gamma_{(j)}\left(y_{i}^{\sigma}\right),
$$

which is equivalent to $\Gamma_{i j}^{\sigma}=\Gamma_{j i}^{\sigma}$. But this is an identity due to the symmetry, and is hence redundant. It follows that the integrability conditions in fibered coordinates reduce to just the "differential conditions" for the functions $F$, and these are the conditions for the curvature of the connection to vanish. It should, however, be noted that, as equations for the unknown functions $F$, these conditions are more complicated than the "differential" integrability conditions in Legendre coordinates.

\section{Lagrangian-Hamiltonian duality}

Our aim now is to establish a duality relationship between the "Lagrangian side" and "Hamiltonian side" for Hamilton-De Donder systems. 


\subsection{From Lagrangian to Hamiltonian}

Consider the affine dual $J^{\dagger} Y \stackrel{\rho}{\rightarrow} J^{*} Y \stackrel{\tau}{\rightarrow} X$ for a fibred manifold $\pi: Y \rightarrow X$ as above, with the canonical $n$-form $\Theta=P \omega_{0}+P_{\sigma}^{i} d y^{\sigma} \wedge \omega_{i}$ and the canonical multisymplectic form $\Omega=d \Theta$ on $J^{\dagger} Y$. On $\pi_{1}: J^{1} Y \rightarrow X$ let a Hamilton-De Donder system be given by a Lagrangian $\lambda$ and its Poincaré-Cartan form $\theta_{\lambda}$. Both the sides of the picture can be related by means of a map Leg: $J^{1} Y \rightarrow J^{\dagger} Y$ fibred over the identity of $Y$, defined by

(5.1) $\operatorname{Leg}^{*} \Theta=\theta_{\lambda}$.

We call Leg an extended Legendre map and equation (5.1) a duality equation. We also construct a composite map leg : $J^{1} Y \rightarrow J^{*} Y$ by setting leg $=\rho \circ$ Leg. This is called the reduced Legendre map.

In local fibred coordinates on $J^{1} Y$ such that $\omega_{0}=d x^{1} \wedge \cdots \wedge d x^{n}$ and related canonical coordinates $\left(x^{i}, y^{\sigma}, P, P_{\sigma}^{j}\right)$ and $\left(x^{i}, y^{\sigma}, P_{\sigma}^{j}\right)$ on $J^{\dagger} X$ and $J^{*} Y$ respectively, the maps Leg and leg are given in coordinates by

$$
P \circ \operatorname{Leg}=L-\frac{\partial L}{\partial y_{j}^{\sigma}} y_{j}^{\sigma}, \quad P_{\sigma}^{j} \circ \operatorname{Leg}=\frac{\partial L}{\partial y_{j}^{\sigma}}
$$

and

$$
P_{\sigma}^{j} \circ \operatorname{leg}=\frac{\partial L}{\partial y_{j}^{\sigma}},
$$

respectively.

If the Lagrangian is regular we can choose local Legendre coordinates on $J^{1} Y$, and then leg is represented by the identity mapping.

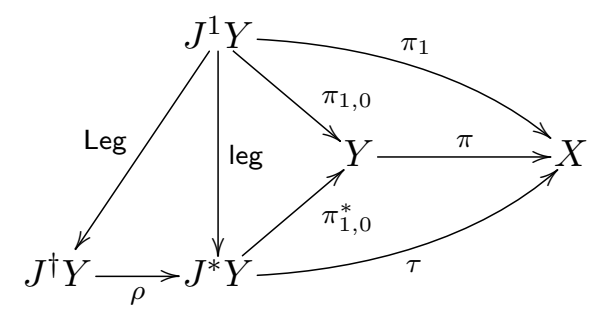

We immediately obtain the following result.

Theorem 5.1. If $\lambda$ is regular then every extended Legendre map is an immersion and every corresponding reduced Legendre map is a local diffeomorphism.

We say that $\lambda$ is hyper-regular if there is an extended Legendre map Leg which is defined globally and which has the property that the corresponding reduced Legendre map leg is a diffeomorphism. Any such map gives rise to a global Hamiltonian section $\mathrm{h}$ by setting $\mathrm{h}=\mathrm{Leg} \circ \mathrm{leg}^{-1}$. If $\lambda$ is regular rather than hyper-regular then we may construct local sections $\mathrm{h}$ in this way. The negative coordinate representative of such a section $\mathrm{h}$ will be the function $\mathcal{H}=-(P \circ \mathrm{h})$, a Hamiltonian function corresponding to $\lambda$.

With help of the definition of the Legendre maps, and using Legendre coordinates, we easily obtain the following results. 
Theorem 5.2. For a hyper-regular Lagrangian $\lambda$ on $J^{1} Y$ the following duality assertions hold:

(1) $\operatorname{leg} \circ \mathcal{H}=H$;

(2) $\left(j^{1} \operatorname{leg}\right)^{*} \Lambda=\tilde{\lambda}$;

(3) leg* $\Omega_{h}=$ leg $^{*} h^{*} \Omega=d \theta_{\lambda}$;

(4) $\operatorname{rank} \mathrm{h}^{*} \Omega=\operatorname{rank} \mathcal{D}_{\mathrm{h}}=\operatorname{rank} d \theta_{\lambda}=\operatorname{rank} \mathcal{D}_{\lambda}=m+n m$;

(5) leg* $\mathcal{D}_{\mathrm{h}}=\mathcal{D}_{\lambda}$;

(6) if $\psi: X \rightarrow J^{*} Y$ is an integral section of $\mathcal{D}_{\mathrm{h}}$ then leg $^{-1} \circ \psi=j^{1} \gamma$ where $\gamma$ is a section of $\pi: Y \rightarrow X$, and furthermore $j^{1} \gamma$ is an integral section of $\mathcal{D}_{\lambda}$;

(7) every integral section of $\mathcal{D}_{\lambda}$ is of the form $j^{1} \gamma$, and $\psi=\operatorname{leg} \circ j^{1} \gamma$ is an integral section of $\mathcal{D}_{\mathrm{h}}$.

\subsection{From Hamiltonian to Lagrangian}

Now let $\Omega_{\mathrm{h}}$ be a Hamiltonian system on the dual jet bundle $\tau: J^{*} Y \rightarrow X$. We have seen that such a system is variational, in that there is a Lagrangian form $\Lambda_{\mathrm{h}}=\mathcal{L}_{\mathrm{h}} \omega_{0}$ whose extremals $\psi$ are precisely the solutions of the Hamilton-De Donder system $\mathcal{D}_{\mathrm{h}}$. But $\Lambda_{\mathrm{h}}$ is a form on $J^{1} J^{*} Y$; we now wish to see if there is, at least locally, a Lagrangian form on the original jet manifold $J^{1} Y$ whose extremals are related to the solutions $\psi$.

Let $U \subset J^{*} Y$ be the domain of a chart $\left(x^{i}, y^{\sigma}, P_{\sigma}^{i}\right)$ contained within the domain of h, and define a map heg : $U \rightarrow J^{1} Y$ fibred over the identity on $Y$ by

$$
y_{i}^{\sigma} \circ \text { heg }=\frac{\partial \mathcal{H}}{\partial P_{\sigma}^{i}} .
$$

If heg is a local diffeomorphism, so that $\mathcal{H}$ satisfies the regularity condition

$$
\operatorname{det}\left(\frac{\partial^{2} \mathcal{H}}{\partial P_{\sigma}^{i} \partial P_{\nu}^{j}}\right) \neq 0
$$

we may construct a local Lagrangian $\lambda=L \omega_{0}$ on $J^{1} Y$ by setting

(5.4) $\lambda=\mathrm{h}\left(\theta_{\lambda}\right) \quad$ where $\quad \theta_{\lambda}=\left(\text { heg }^{-1}\right)^{*} \Theta_{\mathrm{h}}$,

so that

$$
L \circ \text { heg }=-\mathcal{H}+\frac{\partial \mathcal{H}}{\partial P_{\sigma}^{i}} P_{\sigma}^{i}
$$

Theorem 5.3. Let $\Omega_{\mathrm{h}}$ be a Hamilton-De Donder system on $J^{*} Y$ satisfying the regularity condition (5.3) on $U$. The local Lagrangian $\lambda=L \omega_{0}$ (5.5) is then regular and satisfies leg $=$ heg $^{-1}$; furthermore, the solutions of the Euler-Lagrange equations for $\lambda$ are in bijective correspondence with the Hamilton-De Donder extremals of $\Omega_{\mathrm{h}}$. 
Proof. From (5.5) we see that

$$
\frac{\partial}{\partial P_{\nu}^{j}}(L \circ \text { heg })=\frac{\partial^{2} \mathcal{H}}{\partial P_{\sigma}^{i} \partial P_{\nu}^{j}} P_{\sigma}^{i},
$$

whereas a direct calculation using the chain rule gives

$$
\frac{\partial}{\partial P_{\nu}^{j}}(L \circ \text { heg })=\left(\frac{\partial L}{\partial y_{i}^{\sigma}} \circ \text { heg }\right) \frac{\partial}{\partial P_{\nu}^{j}}\left(y_{i}^{\sigma} \circ \text { heg }\right)=\left(\frac{\partial L}{\partial y_{i}^{\sigma}} \circ \text { heg }\right) \frac{\partial^{2} \mathcal{H}}{\partial P_{\sigma}^{i} \partial P_{\nu}^{j}} .
$$

It then follows by regularity of $\Omega_{\mathrm{h}}$ that

$$
P_{\sigma}^{i} \circ \text { heg }^{-1}=\frac{\partial L}{\partial y_{i}^{\sigma}}=P_{\sigma}^{i} \circ \text { leg },
$$

so that leg $=$ heg $^{-1}$ is a local diffeomorphism.

We now observe from (5.5) that

$$
\mathcal{H} \circ \operatorname{leg}=-L+\left(\frac{\partial \mathcal{H}}{\partial P_{\sigma}^{i}} \circ \operatorname{leg}\right)\left(P_{\sigma}^{i} \circ \operatorname{leg}\right)=-L+y_{i}^{\sigma} \frac{\partial L}{\partial y_{i}^{\sigma}}
$$

so that $\mathcal{H}$ is the Hamiltonian function associated classically with the Lagrangian function $L$. Any extremal $\psi$ of the Hamilton-De Donder system $\Omega_{\mathrm{h}}$ thus satisfies the condition that heg $\circ \psi=j^{1} \gamma$ for some extremal $\gamma$ of $L$, and conversely if $\gamma$ is an extremal of $L$ then leg $\circ j^{1} \gamma$ is an extremal of $\Omega_{\mathrm{h}}$.

\subsection{The inverse problem}

We remark finally that this procedure may be applied also to a family of first-order equations

(5.6) $\frac{\partial y^{\sigma}}{\partial x^{i}}-F_{i}^{\sigma}=0, \quad \frac{\partial P_{\sigma}^{i}}{\partial x^{i}}+G_{\sigma}=0$

where $F_{i}^{\sigma}, G_{\sigma}$ are functions defined locally on $J^{*} Y$; such functions may, for example, be obtained from an Ehresmann connection $\Gamma$ on $\tau: J^{*} Y \rightarrow X$ where $F_{i}^{\sigma}=y_{\mid i}^{\sigma} \circ \Gamma$ and $G_{\sigma}=-P_{\sigma \mid i}^{i} \circ \Gamma$. Writing $E$ for the $(n+1)$-form

$$
E=\left(G_{\sigma}+P_{\sigma \mid i}^{i}\right) d y^{\sigma} \wedge \omega_{0}+\left(-F_{i}^{\sigma}+y_{\mid i}^{\sigma}\right) d P_{\sigma}^{i} \wedge \omega_{0}
$$

we see that Corollary 2.4 can be refined as follows.

Theorem 5.4. A system of first order PDEs (5.6) is a Hamilton-De Donder system, equivalent to the Euler-Lagrange equations of a (local) regular Lagrangian $L$, if and only if

$$
\frac{\partial G_{\sigma}}{\partial y^{\nu}}=\frac{\partial G_{\nu}}{\partial y^{\sigma}}, \quad \frac{\partial F_{i}^{\sigma}}{\partial P_{\nu}^{k}}=\frac{\partial F_{k}^{\nu}}{\partial P_{\sigma}^{i}}, \quad \frac{\partial G_{\sigma}}{\partial P_{\nu}^{i}}=\frac{\partial F_{i}^{\nu}}{\partial y^{\sigma}}
$$


and

$$
\operatorname{det}\left(\frac{\partial F_{i}^{\sigma}}{\partial P_{\nu}^{i}}\right) \neq 0
$$

If these conditions hold then

$$
L=F_{i}^{\sigma} P_{\sigma}^{i}+y^{\sigma} \int_{0}^{1}\left(G_{\sigma} \circ \chi\right) d u+P_{\sigma}^{j} \int_{0}^{1}\left(F_{j}^{\sigma} \circ \chi\right) d u,
$$

and the inverse Legendre transformation (giving "velocities" $y_{j}^{\sigma}$ ) is defined by the equations $y_{j}^{\sigma}=F_{j}^{\sigma}$.

Acknowledgments. Research supported by grant GA 201/09/0981 of the Czech Science Foundation and by the IRSES project GEOMECH (EU FP7, nr 246981).

\section{References}

[1] F. Cantrijn, L.A. Ibort and M. De Leon, Hamiltonian structures on multisymplectic manifolds, Rend. Sem. Mat. Univ. Pol. Torino 54 (1996) 225-236.

[2] F. Cantrijn, L.A. Ibort and M. De Leon, On the geometry of multisymplectic manifolds, J. Austral. Math. Soc. (Series A) 66 (1999) 303-330.

[3] J.F. Cariñena, M. Crampin and L.A. Ibort, On the multisymplectic formalism for first order field theories, Diff. Geom. Appl. 1 (1991) 345-374.

[4] Th. De Donder, Théorie Invariantive du Calcul des Variations, Gauthier-Villars, Paris, 1930.

[5] M. de León, J.C. Marrero and D.M. de Diego, A new geometric setting for classical field theories, Banach Center Publications 59 (2003) 189-209.

[6] A. Echeverria-Enriquez, M.C. Muñoz-Lecanda, and N. Román-Roy, Geometry of multisymplectic Hamiltonian first-order field theories, J. Math. Phys. 41 (2000) $7402-7444$.

[7] G. Giachetta, L. Mangiarotti and G. Sardanashvily, New Lagrangian and Hamiltonian Methods in Field Theory, World Scientific, Singapore, 1997.

[8] H. Goldschmidt and S. Sternberg, The Hamilton-Cartan formalism in the calculus of variations, Ann. Inst. Fourier 23 (1973) 203-267.

[9] M.J. Gotay, A multisymplectic framework for classical field theory and the calculus of variations, I. Covariant Hamiltonian formalism, in: Mechanics, Analysis and Geometry: 200 Years After Lagrange, M. Francaviglia and D. D. Holm, eds. (North Holland, Amsterdam, 1990) 203-235. 
[10] K. Grabowska, The Tulczyjew triple for classical fields, arXiv:1109.2533v2 [math.DG] 21 Nov 2011; J. Phys. A 45 (2012) 145207 (35pp).

[11] J. Kijowski and W. Szczyrba, Multisymplectic manifolds and the geometrical construction of the Poisson brackets in the classical field theory, in: Géométrie symplectique et physique mathématique, Coll. Int. C.N.R.S. No 237 (C.N.R.S., 1975) $347-378$.

[12] D. Krupka and O. Štěpánková, On the Hamilton form in second order calculus of variations, in: Geometry and Physics, Proc. Internat. Meeting, Florence, 1982, M. Modugno, ed. (Pitagora, Bologna, 1983) 85-102.

[13] O. Krupková and D.J. Saunders, Affine duality and Lagrangian and Hamiltonian systems, Int. J. Geom. Met. Mod. Phys. 8 (3) (2011) 669-697.

[14] O. Rossi and D. Saunders, Lagrangian and Hamiltonian duality, J. Math. Sci., to appear.

[15] D.J. Saunders, The Geometry of Jet Bundles, London Math. Soc. Lecture Notes Series 142, Cambridge Univ. Press, Cambridge, 1989. 